\title{
Modelado Cinético de la Disolución de Hidroboracita en Acido Clorhídrico
}

\author{
Graciela V. Morales, Oscar D. Quiroga, Ebelia N. Tolaba \\ Facultad de Ingeniería, Consejo de Investigación, INIQUI-CONICET, Universidad Nacional de Salta, \\ Avenida Bolivia 5150, Salta-Argentina (e-mail: gmorales@unsa.edu.ar)
}

Recibido Nov. 04, 2009; Aceptado Ene. 04, 2010; Versión Final recibida Feb. 18, 2010

\begin{abstract}
Resumen
Se realizó el modelado cinético de la disolución de hidroboracita en soluciones de ácido clorhídrico. La disolución se llevó a cabo en un reactor tanque agitado discontinuo a presión atmosférica. Se investigó el efecto de la temperatura, el tamaño de partícula, la relación sólido/líquido, y la concentración del ácido sobre la velocidad de disolución del mineral. Se observa que la velocidad de disolución es muy rápida en todos los casos estudiados, aumenta con el incremento de la temperatura y la disminución del tamaño de partícula, y disminuye con el incremento de la relación sólido/líquido. Los datos experimentales fueron analizados por distintos modelos cinéticos, desarrollados en la literatura, para las transformaciones fluido-sólido reactivo. El modelo cinético mas probable permite inferir que el proceso de disolución ocurre con control difusivo, siendo la energía de activación $13.48 \mathrm{KJ} / \mathrm{mol}$.
\end{abstract}

Palabras clave: hidroboracita, ácido bórico, disolución, cinética, modelado

\section{Kinetics Modeling of Hydroboracite Dissolution in Hydrochloric Acid}

\begin{abstract}
The kinetics modeling of hydroboracite in hydrochloric acid solutions was developed. The dissolution was carried out in a batch reactor at atmospheric pressure. The effects of the temperature, particle size, solid/liquid ratio and concentration of the solution on the dissolution rate were investigated. It was found that the dissolution rate is always very fast, increases with increasing reaction temperature and with decreasing particle size, and decreases with an increase in the solid/liquid ratio. The experimental data were analyzed with different kinetics models presented in literature, for fluid-solid reactive transformations. It was determined that the dissolution rate fit to the diffusive control model, with activation energy of $13.48 \mathrm{KJ} / \mathrm{mol}$.
\end{abstract}

Keywords: hydroboracite, boric acid, dissolution kinetics, modeling 


\section{INTRODUCCION}

La provincia de Salta (Argentina) ocupa el primer lugar internacional en producción y exportación de hidroboracita, mineral que se explota en los yacimientos de la sierra de Sijes, en la Puna salteña. La hidroboracita es un borato de calcio y magnesio hexahidratado $\left(\mathrm{CaO} \cdot \mathrm{MgO} \cdot 3 \mathrm{~B}_{2} \mathrm{O}_{3} \cdot 6 \mathrm{H}_{2} \mathrm{O}\right)$, es insoluble en agua fría y caliente, pero se disuelve en agua acidificada. Sus usos están relacionados con la industria de los fertilizantes y otros compuestos para la agricultura, también se aplica en preparados de cerámicas especiales y como fundente en hornos metalúrgicos para la reducción de menas de titanio.

Tunç et al. (1999), estudiaron la disolución de ulexita en $\mathrm{H}_{2} \mathrm{SO}_{4}$ y $\mathrm{HCl}$ y determinaron que la velocidad de disolución es mayor en $\mathrm{HCl}$ que en $\mathrm{H}_{2} \mathrm{SO}_{4}$ debido a que, en este último caso, la película de producto que se forma sobre la superficie de mineral afecta la velocidad de disolución. Por otra parte, Morales et al. (2000), estudiaron la disolución de hidroboracita en agua con dióxido de carbono disuelto; observaron que el aumento de la temperatura favorece la velocidad de disolución y que la misma se halla influenciada por fenómenos de transporte de masa de reactivos y/o productos. Künkül et al. (2003), estudiaron la disolución de ulexita en soluciones de sulfato de amonio y determinaron que la misma es controlada también por mecanismos difusivos. Alkan et al. (2004), estudiaron los mecanismos cinéticos de la disolución de ulexita original y calcinada en soluciones de ácido oxálico y determinaron que la velocidad de disolución es controlada por la difusión en la capa de producto. Por otra parte, Kurbas et al. (2006) estudiaron la disolución de colemanita en soluciones acuosas de $\mathrm{SO}_{2}$ y ácido bórico; determinaron que la velocidad de transformación del mineral es bien correlacionada por el modelo de Avrami. Demirkiran y Künkül (2007), investigaron la disolución de ulexita en soluciones de ácido perclórico; determinaron que la velocidad de disolución puede ser descripta, también, por el modelo de Avrami. Demirkiran (2008) estudió la cinética de disolución de ulexita en soluciones de acetato de amonio y Demirkiran (2009) investigó la disolución de ulexita en soluciones de nitrato de amonio; determinando que, en ambos casos, la reacción ocurre con control químico. Por otra parte, Ekmekyapar et al. (2008) estudiaron la cinética de disolución de ulexita en soluciones de ácido acético demostrando que la misma obedece al modelo del frente móvil, y Tuba et al. (2009) investigaron la cinética de reacción entre ulexita y ácido fosfórico demostrando que la velocidad de disolución es controlada por el proceso difusivo en la capa de producto que rodea al mineral.

En este trabajo se estudia la disolución de hidroboracita en soluciones de $\mathrm{HCl}$, en un reactor tanque agitado discontinuo a presión atmosférica. Se correlacionan los datos experimentales con distintos modelos cinéticos, desarrollados en la literatura para las transformaciones fluido-sólido reactivo, y selecciona el modelo mas probable para la transformación estudiada.

\section{PARTE EXPERIMENTAL}

Los experimentos de disolución de hidroboracita, se llevaron a cabo en un reactor de tipo tanque agitado discontinuo, de acero inoxidable, a escala laboratorio. La tapa del reactor posee un orificio central por donde se introduce el eje de un agitador mecánico, un orificio descentralizado, que se utiliza para cargar la muestra de mineral y un tubo soldado que se utiliza para tomar las muestras de la fase líquida. Durante la experimentación el reactor se introdujo en un baño termostático con temperatura controlada y se mantuvo el medio agitado mecánicamente. Los experimentos fueron programados sobre la base de un diseño factorial a dos niveles, habiéndose seleccionado como factores (variables independientes) a los siguientes: $T$ (temperatura), $d_{p}$ (diámetro de partícula); Ra (relación masa de sólido a volumen de líquido) y $\mathrm{C}_{\mathrm{A}}$ (concentración del ácido). En la Tabla 1 se muestran los niveles de los factores usados en los experimentos.

Tabla 1: Factores y sus valores usados en los experimentos

\begin{tabular}{|l|r|}
\hline Tamaño de partícula $(\mathrm{mm}) ; \mathrm{d}_{\mathrm{p}}$ & $0.754 ; 1.2$ \\
Temperatura $\left({ }^{\circ} \mathrm{C}\right): \mathrm{T}$ & $30 ; 40$ \\
Sólido/líquido $(\mathrm{g}(\mathrm{S}) / \mathrm{ml}(\mathrm{L})): \mathrm{Ra}$ & $0.01 ; 0.02$ \\
Concentración del ácido $(\mathrm{N}): \mathrm{C}_{\mathrm{A}}$ & $0.25 ; 0.5$ \\
\hline
\end{tabular}


Se mantuvo la velocidad de agitación constante e igual a 520 rpm después de verificar que la misma no tiene efectos significativos sobre la velocidad de disolución del mineral. Se eligió como única respuesta del sistema al grado de transformación que experimenta el reactivo sólido con respecto de la masa de $\mathrm{B}_{2} \mathrm{O}_{3}$, y se la definió como:

$$
X=\frac{M_{B}^{\circ}-M_{B}}{M_{B}^{\circ}}
$$

$M_{B}^{O}$ es la masa inicial de $\mathrm{B}_{2} \mathrm{O}_{3}$ asociada a la partícula y $\mathrm{M}_{\mathrm{B}}$ es la masa asociada en un tiempo dado.

A tiempos de reacción previamente programados se tomaron muestras de la mezcla reaccionante, las cuales fueron inmediatamente filtradas. Del líquido filtrado se tomó una alícuota y se la diluyó con agua destilada. La solución diluida se analizó mediante volumetría.

\section{RESULTADOS Y DISCUSION}

Las reacciones de hidroboracita con $\mathrm{HCl}$ son:

$$
\begin{aligned}
& \mathrm{HCl}_{(\mathrm{ac})}+\mathrm{H}_{2} \mathrm{O} \rightarrow \mathrm{Cl}_{(\mathrm{ac})}^{-}+\mathrm{H}_{3} \mathrm{O}_{(\mathrm{ac})}^{+} \\
& \text {CaO.MgO. } 3 \mathrm{~B}_{2} \mathrm{O}_{3} \cdot 6 \mathrm{H}_{2} \mathrm{O}_{(\mathrm{s})}+4 \mathrm{Cl}_{(\mathrm{ac})}^{-}+4 \mathrm{H}_{3} \mathrm{O}^{+}{ }_{(\mathrm{ac})} \rightarrow 6 \mathrm{H}_{3} \mathrm{BO}_{3(\mathrm{ac})}+\mathrm{CaCl}_{2(\mathrm{ac})} \\
& +\mathrm{MgCl}_{2(\mathrm{ac})}+3 \mathrm{H}_{2} \mathrm{O}
\end{aligned}
$$

En la reacción (3) se forman cloruro de calcio y cloruro de magnesio que, por su solubilidad, permanecen en solución. La fotografía de microscopia electrónica SEM (Fig.1), muestra la formación de tres productos diferentes: los cristales de masas mas pequeñas corresponden a $\mathrm{BO}_{3} \mathrm{H}_{3}$, los cristales en forma de bastón corresponden a $\mathrm{CaCl}_{2}$ y los de forma rectangular $\mathrm{MgCl}_{2}$. Esta fotografía, confirman la ocurrencia de la reacción (3).

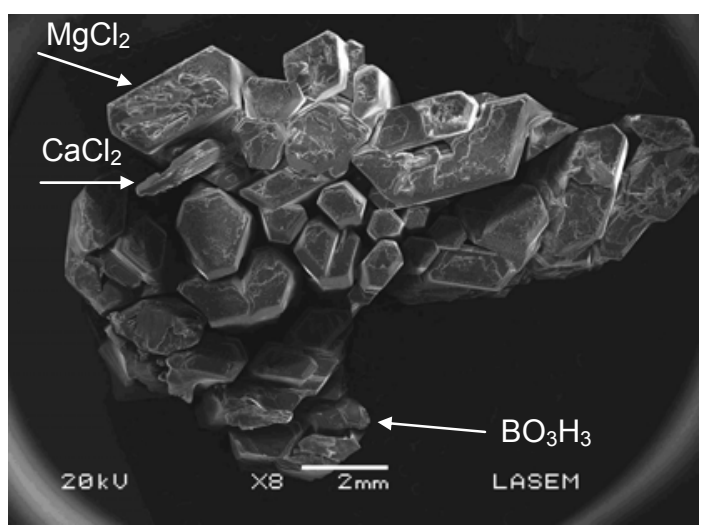

Fig. 1: Fotografía SEM: productos de disolución de hidroboracita en $\mathrm{HCl}$

En la Fig. 2 se presenta la influencia del tamaño de partícula en la disolución de hidroboracita en $\mathrm{HCl}$ $(0.5 \mathrm{~N})$ a $40{ }^{\circ} \mathrm{C}$ y con la relación sólido/líquido $0.01 \mathrm{~g}(\mathrm{~S}) / \mathrm{ml}(\mathrm{L})$. Se observa que la velocidad de disolución aumenta con la disminución del tamaño de partícula del mineral. Este mismo efecto se manifiesta a otras temperaturas de disolución.

Por otra parte, en la Fig. 3, se presentan los resultados experimentales de la disolución de hidroboracita en $\mathrm{HCl}(0.5 \mathrm{~N})$ a $30{ }^{\circ} \mathrm{C}$ y $40{ }^{\circ} \mathrm{C}$, para un diámetro de partícula $1.2 \mathrm{~mm}$ y una relación sólido/ líquido $0.01 \mathrm{~g}(\mathrm{~S}) / \mathrm{ml}(\mathrm{L})$. Se observa que a medida que aumenta la temperatura aumenta la velocidad de disolución del mineral. Así para un incremento de $10^{\circ} \mathrm{C}$ en la temperatura, el grado de disociación del mineral se incrementa en un $23 \%$ a los 60 segundos comenzada la disolución. 


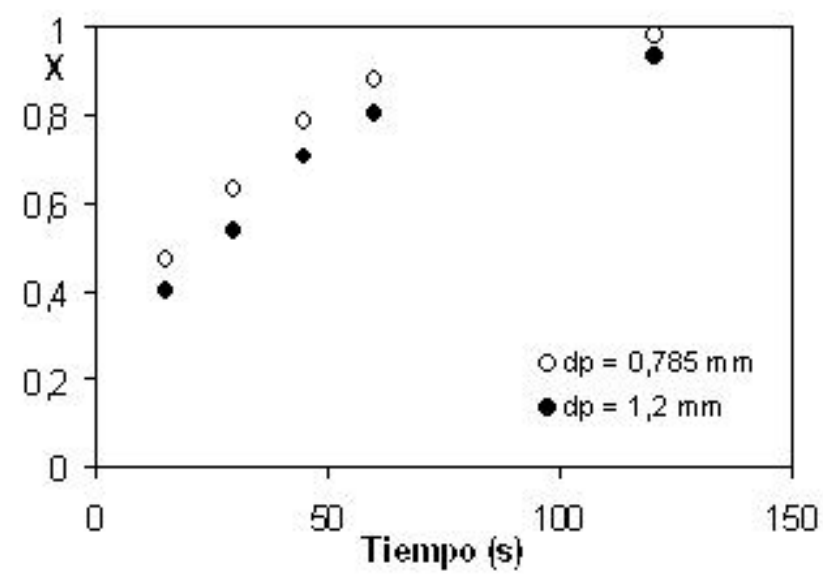

Fig. 2: Influencia del diámetro de partícula en la disolución de hidroboracita

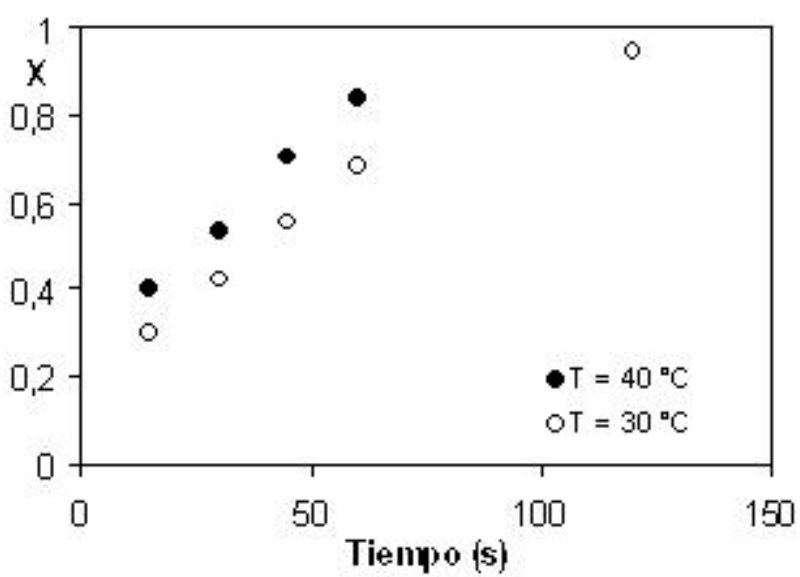

Fig. 3: Influencia de la temperatura en la disolución de hidroboracita

Se realizaron, también, experimentos para investigar el efecto de la relación sólido/líquido en la disolución de hidroboracita en $\mathrm{HCl}(0.5 \mathrm{~N})$. Se presentan datos a $40^{\circ} \mathrm{C}$ y diámetro de partícula 1.2 $\mathrm{mm}$ (Fig. 4). Se observa que un este efecto es menos significativo que los anteriores dado que un incremento del doble en la relación sólido/líquido, hizo disminuir el grado de transformación del mineral únicamente en un $11 \%$ a los 120 segundos.

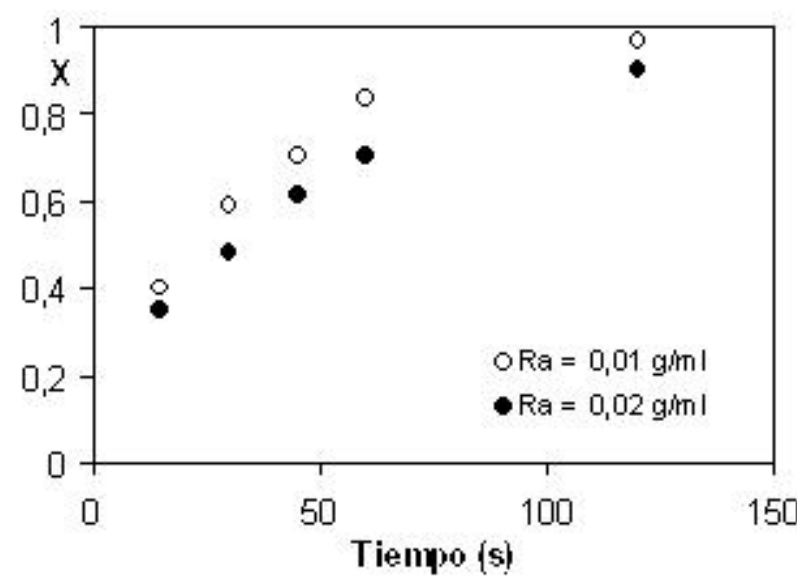

Fig. 4: Influencia de la relación sólido/líquido en la disolución de hidroboracita

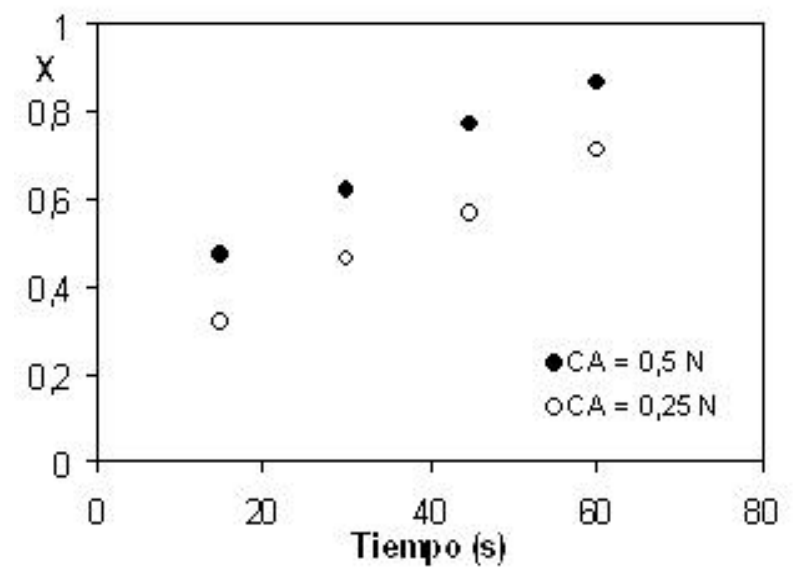

Fig. 5: Influencia de concentración del ácido en la disolución de hidroboracita

Información Tecnológica Vol. - 21 Nº 5 - 2010 
La velocidad de disolución del mineral aumenta siempre con el incremento de la concentración del ácido; por ejemplo aumentando la concentración al doble, a $40{ }^{\circ} \mathrm{C}$ y diámetro de partícula $1.2 \mathrm{~mm}$, el grado de transformación del mineral aumenta el $22 \%$ a los 60 segundos de reacción (Fig. 5).

\section{TRATAMIENTO CINETICO DE DATOS EXPERIMENTALES}

En términos generales se sabe que la velocidad inicial observada, $\mathbf{r}_{\mathbf{S}}^{\mathbf{o}}$, de una transformación fluidosólido reactivo dada, depende de numerosos factores como por ejemplo: la temperatura, $\mathrm{T}$, el diámetro de partícula, $\mathrm{d}_{\mathrm{p}}$, la concentración del reactivo fluido, $\mathrm{C}_{\mathrm{A}}$ y la relación sólido/líquido, $\mathrm{Ra}$. La influencia que tienen estos factores del sistema experimental en la velocidad inicial observada del proceso, puede resumirse cuantitativamente mediante la ecuación siguiente (Quiroga et al., 2000):

$$
r_{S}^{o}=k^{o} \exp (-E / R T) C_{A}^{n} d_{p}^{f} R a^{h}
$$

$k^{\circ}$ es el coeficiente factor de frecuencia, $E$ es el coeficiente energía de activación aparente y $n, f$ y $h$ son coeficientes exponenciales. Estos coeficientes se estiman a partir de datos de la velocidad inicial calculados para cada uno de los niveles de los factores. Si bien es cierto que la ec. (4) no es totalmente exacta, a través de las estimaciones de sus coeficientes es posible inferir cuál es la etapa controlante del proceso.

Para el tratamiento cinético de los datos experimentales de las disoluciones de hidroboracita en $\mathrm{HCl}$, se desarrolló un software que permite correlacionar los datos cinéticos mediante distintos modelos que se presentan en la literatura, para la disolución de sistemas heterogéneos fluido-sólido reactivo (Quiroga et al., 2000). El software desarrollado tiene como propósito principal resolver, para cada uno de los modelos, la siguiente función objetivo:

$$
\phi_{m}=\operatorname{Min}_{b_{m}}\left\{\sum_{j=1}^{N e x}\left(X_{m, j}-X_{e x, j}\right)^{2}\right\}
$$

$\mathrm{X}_{\mathrm{ex}, \mathrm{j}}$ es la conversión medida en el j-ésimo experimento; $\mathrm{X}_{\mathrm{m}, \mathrm{j}}$ es la conversión que predice el $\mathrm{m}$ ésimo modelo en el j-ésimo experimento; $b_{m}=\left(b_{1}, b_{2}, b_{3}, \ldots\right)^{\top}$, vector de los coeficientes adjuntos al m-ésimo modelo; $\phi_{\mathrm{m}}$ función objetivo adjunta al m-ésimo modelo, cuyo mínimo debe estimarse por la oportuna elección de $b_{m}$. Una vez que los datos del sistema experimental han sido correlacionados por los distintos modelos cinéticos, sobre la base de la menor varianza asociada a cada modelo, el software presenta un listado de los modelos más probables. Una segunda discriminación de modelos es realizada a través de las estimas de los coeficientes cinéticos adjuntos a cada uno de los modelos más probables. En la Tabla 2 se resumen los datos correspondientes al tratamiento cinético de la disolución de hidroboracita en $\mathrm{HCl}$ y se presenta el modelo cinético más probable, que es un modelo semiempírico de control difusional.

Tabla 2: Tratamiento cinético de la disolución de hidroboracita

Velocidad de reacción inicial:

$r_{S}^{o}=3.96 \exp \left(\frac{-1621}{T}\right) d_{p}^{0.068} \frac{C_{A}^{0.807}}{R^{0.228}} \quad E=3209.58 \mathrm{cal} / \mathrm{mol}=13.48 \mathrm{~kJ} / \mathrm{mol}$

Modelo de Control Difusional: $\quad X=1-\left(1-b_{1} t^{b_{2}}\right)^{3} \quad$ con: $\quad 0.68<b_{2}<0.83 \quad 0.015<b_{1}<0.027$

Cuando el parámetro $b_{2}$ del modelo cinético es $0<b_{2}<1$ se define $b_{1}=\frac{2 b m_{B} C_{A}\left(D_{E} / \pi\right)^{b_{2}}}{a \rho b_{2} d_{p}}$, siendo 
a y b los coeficientes estequiométricos del $\mathrm{HCl}$ y la hidroboracita, respectivamente, ec. (3); $\mathrm{D}_{\mathrm{E}}$ la difusividad efectiva, $m_{B}$ el peso molecular de la hidroboracita y $\rho$ la densidad de la hidroboracita. En la Fig. 6 se presenta el ajuste entre los valores de conversión experimental $\left(X_{\text {ex }}\right)$ y los valores predichos por el modelo cinético $\left(\mathrm{X}_{\mathrm{m}}\right)$. Puede observase que el ajuste es bastante bueno.

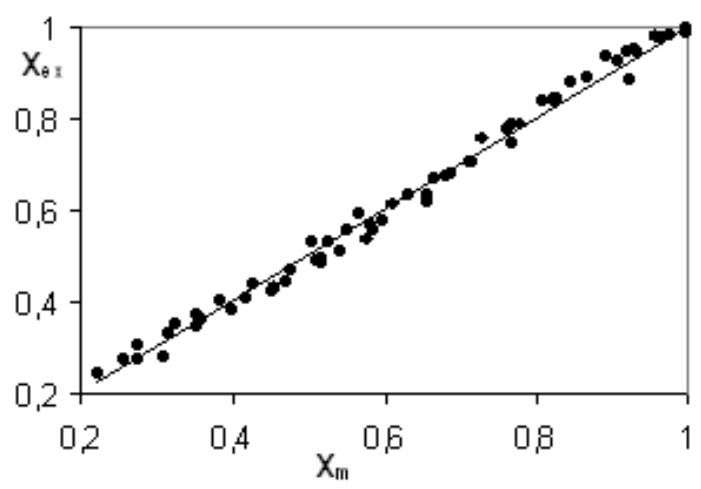

Fig. 6: Ajuste entre los valores de conversión experimental y los valores predichos por el Modelo

\section{CONCLUSIONES}

Se observa que la velocidad de disolución de hidroboracita en $\mathrm{HCl}$ es muy rápida, aumenta con el incremento de la temperatura y de la concentración del ácido, y disminuye con el incremento del diámetro de partícula y de la relación sólido-líquido; siendo este último efecto el menos significativo. Dado que es posible despreciar el efecto del diámetro de partícula sobre la velocidad de reacción inicial (Tabla 2) y que el valor de la energía de activación aparente calculado es bajo (13.48 kJ/mol); es posible inferir que el proceso de disolución ocurre con control difusivo. Estas observaciones apoyan el modelo cinético encontrado, que es un modelo de control difusional.

\section{REFERENCIAS}

Alkan, M., M. Dogan y H. Namli, Dissolution kinetics and mechanism of ulexite in oxalic acid solutions, Ind. Eng. Chem. Res., 43, 1591-1598 (2004).

Demirkiran, N. y A. Künkül, Dissolution kinetics of ulexite in perchloric acid solutions, Int. J. Miner. Process 83, 76-80 (2007).

Demirkiran, N., A study on dissolution of ulexite in ammonium acetate solutions, Chemical Engineering Journal 141, 180-186 (2008).

Demirkiran, N., Dissolution kinetics of ulexite in ammonium nitrate solutions, Hydrometallurgy 95, 198202 (2009).

Ekmekyapar, A., N. Demirkiran y A. Künkül, Dissolution kinetics of ulexite in acetic acid solutions, Chemical Engineering Research and Design, 86, 1101-1016 (2008).

Künkül, A., N. Dermirkiran y A. Baysar, Dissolution kinetics of ulexite in ammonium sulfate solutions, Ind. Eng. Chem. Res., 42,982-986 (2003).

Kurbas, A., M.M. Kocakerim, O. Kucuk y A. Yartasi, Dissolution of colemanite in aqueous solutions saturated with both sulfur dioxide $\left(\mathrm{SO}_{2}\right)$ and boric acid, Ind. Eng. Chem. Res., 45, 1857-1862 (2006).

Morales, G.V., M. E. Capretto, L. Mercado Fuentes y O.D. Quiroga, Dissolution kinetics of hydroboracite in water saturated with carbon dioxide, Hydrometallurgy, 58, 127-133 (2000).

Quiroga, O. D., G.V. Morales, L.T. Villa y M.S. Vicente, Tratamiento cinético de reacciones fluidosólido reactivo. II) Desarrollo de un software y ejemplo de aplicación, JATRAMI, 405 - 410 (2000).

Tuba, H. y A. Yartasi, Kinetic investigation of reaction between ulexite ore and phosphoric acid, Hydrometallurgy 96, 294-299 (2009).

Tunç, M., M.M. Kocakerim, S. Yapici y Y.S. Bayrakceken, Dissolution of ulexite in $\mathrm{H}_{2} \mathrm{SO}_{4}$ solution, Hydrometallurgy: 51, 359-370 (1999). 